\title{
Qualidade da atenção primária à saúde no Brasil e associação com o Programa Mais Médicos
}

\author{
Milena Rodrigues Agostinho Rech, ${ }^{1}$ Lisiane Hauser, ${ }^{1}$ Lucas Wollmann, ${ }^{1}$ Rudi \\ Roman, ${ }^{1}$ Sotero Serrate Mengue, ${ }^{1}$ Elisandrea Sguario Kemper, ${ }^{2}$ Alexandre de \\ Souza Ramos Florencio, ${ }^{2}$ Gerardo Alfaro, ${ }^{2}$ Renato Tasca ${ }^{2}$ e Erno Harzheim ${ }^{1}$
}

Como citar Rech MRA, Hauser L, Wollmann L, Roman R, Mengue SS, Kemper SE, et al. Qualidade da atenção primária no Brasil e associação com o Programa Mais Médicos. Rev Panam Salud Publica. 2018;42:e164. https://doi.org/10.26633/RPSP.2018.164

RESUMO Objetivo. Avaliar a qualidade da atenção primária à saúde (APS) no Brasil e sua associação com o Programa Mais Médicos (PMM).

Métodos. Este estudo transversal de abrangência nacional utilizou a ferramenta PCATool-Brasil para avaliar a qualidade da APS a partir da experiência dos usuários vinculados a três categorias de médicos: médicos brasileiros do PMM, médicos cubanos do PMM e médicos brasileiros não vinculados ao PMM. Os seguintes escores foram calculados: Escore Geral da APS, Escore de Acesso e Escore de Longitudinalidade. A associação entre o Escore Geral obtido, a categoria do médico e outras características dos usuários e dos profissionais foi investigada por análise multinível.

Resultados. O Escore Geral da APS para o Brasil foi 6,78, e o Escore de Longitudinalidade, 7,43. Não houve diferença entre esses escores para as três categorias de médicos. O Escore de Acesso para o Brasil foi de 4,24, havendo diferença pequena, mas significativa (P-valor <0,001), entre as categorias de médicos: médicos cubanos do PMM com 4,43 (IC: 4,32 a 4,54), médicos brasileiros do PMM com 4,08 (IC: 3,98 a 4,18) e médicos brasileiros não vinculados ao PMM com 4,20 (IC: 4,09 a 4,32). Na análise multinível, idade, estrato socioeconômico, presença de doenças crônicas e o fato de o médico realizar visita domiciliar influenciaram positivamente o Escore Geral. Conclusões. O tipo de médico não influenciou o grau de orientação aos atributos da APS (Escore Geral) no Brasil. O PMM associou-se a maiores Escores de Acesso em regiões de maior vulnerabilidade socioeconômica. Com a análise multinível, identificamos que o fortalecimento da APS também pode ser alcançado ao reforçar papéis fundamentais dos médicos que trabalham na APS (como as visitas domiciliares) e aprimorar o acesso das populações de maior vulnerabilidade socioeconômica e de pessoas mais jovens ou sem doenças crônicas.

Palavras-chave Atenção primária à saúde; Estratégia Saúde da Família; pesquisa sobre serviços de saúde; Sistema Único de Saúde; sistemas de saúde; Brasil.

\footnotetext{
Universidade Federal do Rio Grande do Sul (UFRGS), Programa de Pós-Graduação em Epidemiologia, Porto Alegre, RS, Brasil. Correspondência: Milena Rodrigues Agostinho Rech, milena_ags@hotmail.com

2 Organização Pan-Americana de Saúde (OPAS)/ Organização Mundial da Saúde (OMS), Brasília (DF), Brasil.
}

O Sistema Único de Saúde (SUS), sistema público de saúde no Brasil, tem a cobertura universal como princípio e a atenção primária à saúde (APS) como porta de entrada e ordenadora da rede de atenção $(1,2)$. A Estratégia Saúde da
Família (ESF) - principal modelo de APS no SUS - é caracterizada por equipe multiprofissional, responsável por cerca de 3000 usuários vinculados a uma unidade de saúde (1, 2). Em julho de 2016, 123 milhões de brasileiros eram assistidos 
por 40098 equipes de saúde da família (3). Contudo, a literatura mostra heterogeneidade em relação a estrutura, suficiência de médicos e qualidade dos serviços prestados na APS (4-9). Além disso, estudos feitos com o Instrumento de Avaliação da Atenção Primária à Saúde (PCATool-Brasil) mostraram diferenças na qualidade da APS e um grau de orientação baixo para a maioria dos seus atributos, com piores resultados para acesso e integralidade (10-14).

A dificuldade de acesso no Brasil pode ser expressa pelo menor número de consultas médicas no SUS (2,77 consultas / habitante/ano) em comparação a sistemas de países com APS forte (7,6 consultas/habitante/ano na Espanha, 5,9 consultas/habitante/ano no Reino Unido e 7,7 consultas/habitante/ano no Canadá) e pela fragilidade na marcação de consultas ambulatoriais ou atendimentos de situações clínicas emergenciais (15-17). Em parte, o problema do acesso é explicado pela baixa densidade de médicos no SUS (1,95 médico/1 000 habitantes) quando comparado aos sistemas de países com APS forte (3,8/1 000 na Espanha, 2,85/1 000 no Reino Unido e 2,53/1 000 no Canadá). Vale mencionar que há três vezes mais médicos na rede suplementar brasileira, privada, do que no SUS. Além disso, a distribuição desses profissionais no Brasil é heterogênea, variando de 0,98 por 1000 habitantes na região Norte a 2,61 na região Sudeste $(18,19)$.

Instituído no Brasil em 2013, o Programa Mais Médicos (PMM) foi criado para prover médicos para atuar na APS do SUS $(20,21)$. No primeiro ano do programa, 1834 médicos graduados em instituições brasileiras, com cadastro no Conselho Federal de Medicina (CFM), aderiram ao mesmo. As demais vagas foram preenchidas por 1184 intercambistas individuais (médicos estrangeiros e brasileiros formados em instituições estrangeiras, sem revalidação nacional do diploma) e por 11150 médicos cooperados provenientes de Cuba, a partir de cooperação internacional entre Ministério da Saúde e Organização Pan-Americana da Saúde/Organização Mundial da Saúde (OPAS/OMS) (20, 21). Em 2016, existiam 15205 médicos ativos no PMM, dentre os quais 4561 brasileiros vinculados ao CFM, 1790 intercambistas individuais e 8854 cooperados cubanos (22).

$\mathrm{O}$ objetivo do presente estudo foi estimar a qualidade da APS brasileira, medida pela experiência do usuário conforme o Instrumento PCAToolBrasil, e avaliar a sua associação com o provimento de médicos pelo PMM.

\section{MATERIAIS E MÉTODOS}

O presente estudo transversal, de abrangência nacional, foi realizado com usuários adultos (18 anos de idade ou mais) e médicos da ESF das cinco regiões do país. A experiência dos usuários, medida com o Instrumento PCATool-Brasil, foi estimada e comparada em relação a três categorias de médicos: cubano do PMM (grupo MMCuba), brasileiro do PMM (grupo MMBrasil) e brasileiro não pertencente ao PMM (grupo MedESF).

O PCATool-Brasil é um instrumento validado para avaliar a qualidade da APS a partir de questões que medem a presença e a extensão de seus atributos definidores: Acesso de Primeiro Contato, Longitudinalidade, Coordenação, Integralidade, Orientação Familiar, Orientação Comunitária e Competência Cultural. Os atributos podem ser avaliados individualmente, determinando o escore do atributo, ou conjuntamente, determinando o grau de orientação à APS (Escore Geral da APS) (23). O PCATool-Brasil possui uma versão reduzida, validada para usuários, composta por 23 itens que permitem determinar exclusivamente o Escore Geral da APS (24).

O cálculo amostral foi realizado para estimar e comparar o grau de orientação à APS (Escore Geral da APS) na experiência dos usuários e sua associação com os três grupos de médicos. Para isso, assumiu-se uma diferença de 0,3 ponto no Escore Geral da APS entre os grupos (escore varia de 0 a 10), com desvio padrão de 1,7 , poder estatístico de $80 \%$, nível de significância de $5 \%$ e efeito de delineamento (DEFF) de 3,4. Com base nesses parâmetros, a amostra foi estimada em 5161 usuários. Considerando uma perda de $20 \%$, o tamanho de amostra foi estabelecido em 6193 usuários adultos. O total de usuários foi distribuído de forma igual entre os três grupos de médicos, optando-se por entrevistar 12 pacientes por médico, controlando-se o efeito de dependência médico-paciente com estratégias de análise por conglomerados. Foi realizada amostragem sistemática de unidades de saúde da família (um médico por unidade de saúde), com dis- tribuição proporcional à presença de médicos conforme as regiões do país.

Os médicos responderam um questionário com informações sociodemográficas, de formação profissional e de avaliação do PMM. Os usuários responderam dois instrumentos: um questionário composto por variáveis sociodemográficas, de morbidade, de cuidados recebidos na ESF; e o PCAToolBrasil versão reduzida, associado a itens da versão expandida para complementar os componentes Longitudinalidade e Acesso $(23,24)$. Para ser entrevistado, o médico deveria estar há pelo menos 1 ano na equipe de saúde de família selecionada. Por sua vez, os usuários tiveram como condição para a entrevista ter tido ao menos duas consultas com o médico selecionado, sendo uma delas no dia da entrevista.

O cálculo do Escore Geral da APS foi realizado pela média aritmética das respostas dos usuários aos itens que constituem a versão reduzida do PCATool-Brasil (24). O cálculo do Escore de Acesso e do Escore de Longitudinalidade foi obtido pela média aritmética da pontuação obtida nos itens que integram cada um desses componentes (23). Os escores obtidos foram transformados em escala contínua que varia de 0 a 10 , sendo classificados como altos os valores iguais ou superiores a 6,6, conforme ponto de corte definido pelo estudo de validação do instrumento (23).

Na comparação dos grupos de médicos quanto ao Escore de Acesso, Escore de Longitudinalidade e Escore Geral da APS, foi utilizada a análise de variância (ANOVA) com ajuste de Bonferroni para as múltiplas comparações. Considerouse a mesma metodologia para a comparação desses escores quando estratificados por região do Brasil. Para comparar os grupos de médicos quanto à proporção de Alto Escore (maior ou igual a 6,6) atribuída a cada grupo, utilizou-se o teste do qui-quadrado, seguido, quando necessário, do teste de comparação múltipla (25).

As análises foram realizadas utilizando o módulo Complex Samples do PASW versão 18, que possibilitou incorporar a estrutura complexa da amostra, caracterizada pelas regiões do Brasil (estratos) e pela correlação entre as medidas dos usuários para cada médico escolhido para o estudo (conglomerados) (25). Foi considerado um nível de significância de $5 \%$. 
A metodologia de análise multinível foi aplicada para investigar a associação entre o grupo do médico e o Escore Geral da APS, ajustado por características sociodemográficas e de vínculo com o serviço por parte dos usuários e também por aspectos sociodemográficos e de formação dos médicos. O modelo foi constituído por dois níveis: um dos usuários, outro dos médicos. Inicialmente, no modelo multivariável, foram incluídas as características de usuários e de médicos que se mostraram, individualmente, associadas ao Escore Geral da APS com $P<0,20$. Posteriormente, foram excluídas as características com maiores $P$-valores, de forma que o modelo final contempla somente características associadas de forma ajustada ao Escore Geral da APS, com $P \leq 0,05$. Essa análise foi realizada utilizando o procedimento MIXED do SAS versão 9.4 (26). Os resultados foram apresentados pela estimativa do coeficiente beta seguida do seu intervalo de confiança de 95\% (IC95\%) e pelo $P$-valor.

O estudo foi aprovado pelo comitê de ética do Hospital de Clínicas de Porto Alegre (parecer 1.219.926). Todos os entrevistados assinaram um termo de consentimento livre e esclarecido. Os questionários foram aplicados nas unidades de saúde por entrevistadores treinados que utilizaram um tablet. As informações foram transferidas de maneira anônima para análise.

\section{RESULTADOS}

Entre julho e novembro de 2016, foram entrevistados 6160 usuários $(99,5 \%$ da amostra necessária) e 509 médicos das cinco regiões brasileiras. As características de usuários e médicos e sua distribuição de acordo com as três categorias de médicos aparecem na tabela 1 .

A tabela 2 apresenta os resultados da aplicação do Instrumento PCATool-Brasil para o país e conforme as regiões. A percepção dos usuários resultou em um Escore Geral da APS no Brasil de 6,78. Embora sem diferença estatisticamente significativa $(P=0,215)$, o Escore geral da APS no grupo MMCuba foi discretamente superior à média nacional, com valor de 6,86.

TABELA 1. Características sociodemográficas e histórico de saúde e de atendimento de usuários adultos e características sociodemográficas, de formação e trabalho de médicos cubanos e brasileiros participantes ou não do Programa Mais Médicos, Brasil, 2016

\begin{tabular}{|c|c|c|c|c|}
\hline \multirow[b]{2}{*}{ Características } & \multicolumn{4}{|c|}{$\mathrm{n}(\%)$ ou média (ep) a } \\
\hline & $\begin{array}{c}\text { Geral } \\
\mathrm{n}=6160\end{array}$ & $\begin{array}{l}\text { MMCuba }^{\mathrm{b}} \\
\mathrm{n}=2087\end{array}$ & $\begin{array}{c}\text { MMBrasil }^{b} \\
\mathrm{n}=2062\end{array}$ & $\begin{array}{l}\text { MedESF }^{b} \\
\mathrm{n}=2011\end{array}$ \\
\hline \multicolumn{5}{|l|}{ Características sociodemográficas dos usuários } \\
\hline Sexo feminino & $4667(75,8)$ & $1613(77,3)$ & $1550(75,2)$ & $1504(74,8)$ \\
\hline Sexo masculino & $1493(24,2)$ & $474(22,7)$ & $512(24,8)$ & $507(25,2)$ \\
\hline Idade (anos) & $47,8(0,2)$ & $47,0(0,5)$ & $47,9(0,4)$ & $48,5(0,5)$ \\
\hline Branca & $2055(33,4)$ & $721(34,5)$ & $636(30,8)$ & $698(34,7)$ \\
\hline \multicolumn{5}{|l|}{ Região do país onde se localiza a unidade de saúde } \\
\hline Centro-0este & $375(6,1)$ & $132(6,3)$ & $133(6,4)$ & $110(5,5)$ \\
\hline Nordeste & $2194(35,6)$ & $745(35,7)$ & $761(36,9)$ & $688(34,2)$ \\
\hline Norte & $722(11,7)$ & $252(12,1)$ & $245(11,9)$ & $225(11,2)$ \\
\hline Estrato $\mathrm{D}$ e $\mathrm{E}$ & $2632(42,7)$ & $946(45,3)$ & $885(42,9)$ & $801(39,8)$ \\
\hline \multicolumn{5}{|l|}{ Histórico de saúde/ Experiência na unidade de saúde } \\
\hline \multicolumn{5}{|l|}{ Presença de pelo menos uma doença crônica } \\
\hline Sim & $3598(58,5)$ & $1197(57,4)$ & $1213(58,9)$ & $1188(59,1)$ \\
\hline Não & $2556(41,5)$ & $887(42,6)$ & $848(41,1)$ & $821(40,9)$ \\
\hline \multicolumn{5}{|l|}{$\geq 1$ internação hospitalar no último ano } \\
\hline $\operatorname{Sim}$ & $961(15,6)$ & $320(15,3)$ & $320(15,5)$ & $321(16,0)$ \\
\hline Não & $5199(84,4)$ & $1767(84,7)$ & $1742(84,5)$ & $1690(84,0)$ \\
\hline Número de consultas médicas na unidade de saúde no último ano ${ }^{\mathrm{b}}$ & $5,7(0,1)$ & $5,9(0,1)$ & $5,7(0,1)$ & $5,5(0,1)$ \\
\hline \multicolumn{5}{|l|}{ Tipo de consulta no dia da entrevista } \\
\hline Agendada & $3287(53,4)$ & $1005(48,2)$ & $1210(58,7)$ & $1072(53,3)$ \\
\hline
\end{tabular}


TABELA 1. (Continuação)

\begin{tabular}{|c|c|c|c|c|}
\hline \multirow[b]{2}{*}{ Características } & \multicolumn{4}{|c|}{ n (\%) ou média (ep) ${ }^{\mathrm{a}}$} \\
\hline & $\begin{array}{c}\text { Geral } \\
n=6160\end{array}$ & $\begin{array}{l}\text { MMCuba }^{b} \\
n=2087\end{array}$ & $\begin{array}{c}\text { MMBrasil }^{b} \\
n=2062\end{array}$ & $\begin{array}{l}\text { MedESF }^{b} \\
\mathrm{n}=2011\end{array}$ \\
\hline \multicolumn{5}{|c|}{ A unidade fica aberta pelo menos algumas noites de dias úteis até as 20 horas? } \\
\hline Com certeza sim/provavelmente sim & $466(7,5)$ & $147(7,0)$ & $162(7,8)$ & $158(7,8)$ \\
\hline Com certeza não/provavelmente não & $4735(76,9)$ & $1644(78,8)$ & $1556(75,5)$ & $1535(76,4)$ \\
\hline Não sabe/não lembra & $959(15,6)$ & $296(14,2)$ & $345(16,7)$ & $318(15,8)$ \\
\hline \multicolumn{5}{|l|}{0 Dr. realiza visita domiciliar } \\
\hline $\operatorname{Sim}$ & $3777(61,3)$ & $1348(64,6)$ & $1224(59,4)$ & $1205(59,9)$ \\
\hline Não/não sabe & $2383(38,7)$ & $739(35,5)$ & $838(40,6)$ & $806(40,1)$ \\
\hline Características dos Médicos & $n=509$ & $\mathrm{n}=174$ & $\mathrm{n}=173$ & $n=162$ \\
\hline Idade do médico & $40,1(0,5)$ & $41,9(0,6)$ & $33,5(0,8)$ & $45,3(1,1)$ \\
\hline \multicolumn{5}{|l|}{ Sexo do médico } \\
\hline Feminino & $293(57,6)$ & $117(67,2)$ & $90(52,0)$ & $86(53,1)$ \\
\hline Masculino & $216(42,4)$ & $57(32,8)$ & $83(48,0)$ & $76(46,9)$ \\
\hline Tempo (em anos) de formado em medicina & $13,9(0,5)$ & $17,2(0,6)$ & $6,3(0,7)$ & $18,4(1,1)$ \\
\hline \multicolumn{5}{|l|}{ Especialidade do médico } \\
\hline Residência em medicina de família e comunidade & $158(31,0)$ & $136(78,2)$ & $8(4,6)$ & $14(8,6)$ \\
\hline Especialização em medicina de família e comunidade & $137(26,9)$ & $20(11,5)$ & $103(59,5)$ & $14(8,6)$ \\
\hline Outra residência ou especialização & $127(25,0)$ & $17(9,8)$ & $24(13,9)$ & $86(53,1)$ \\
\hline Nenhuma residência ou especialização & $87(17,1)$ & $1(0,6)$ & $38(22,0)$ & $48(29,6)$ \\
\hline \multicolumn{5}{|l|}{ Outro vínculo empregatício } \\
\hline Não & $294(57,8)$ & $172(98,9)$ & $76(43,9)$ & $46(28,4)$ \\
\hline $\operatorname{Sim}$ & $215(42,2)$ & $2(1,1)$ & $97(56,1)$ & $116(71,6)$ \\
\hline Tempo (em anos) de atuação na unidade de saúde & $3,2(0,1)$ & $2,4(0,0)$ & $2,2(0,1)$ & $5,2(0,3)$ \\
\hline \multicolumn{5}{|l|}{ Número médio de atendimentos por turno de 4 horas } \\
\hline Até 12 & $135(26,5)$ & $52(29,9)$ & $56(32,4)$ & $27(16,7)$ \\
\hline 13 a 15 & $139(27,3)$ & $51(29,3)$ & $47(27,2)$ & $41(25,3)$ \\
\hline 16 a 18 & $131(25,7)$ & $48(27,6)$ & $45(26,0)$ & $38(23,5)$ \\
\hline 19 ou mais & $103(20.2)$ & $23(13,2)$ & $25(14,5)$ & $55(34,0)$ \\
\hline
\end{tabular}

a Resultados expressos em n (\%) ou média (erro padrão).

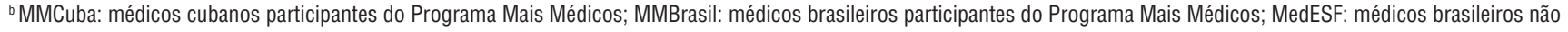
participantes do Programa Mais Médicos.

Foi identificado um alto Escore de Longitudinalidade no país, com valor de 7,43, porém sem diferença significativa entre os grupos (tabela 2). Quanto ao Escore de Acesso, o valor obtido para o Brasil, de 4,24, foi considerado baixo. O Escore de Acesso foi significativamente mais alto no grupo MMCuba (4,43, IC95\%: 4,32 a 4,54) do que os outros grupos (MMBrasil: 4,08, IC95\%: 3,98 a 4,18; MedESF: 4,20, IC95\%: 4,09 a 4,32), apesar da pequena diferença entre os valores dos escores (tabela 2). Na região Nordeste, observou-se diferença estatisticamente significativa para o Escore Geral e para o Escore de Acesso de acordo com tipo de médico. O Escore Geral da APS, na região, foi alto para o grupo MMCuba (6,9 e IC:6,72-7,09), mas muito próximo dos escores do grupo MMBrasil (6,55 e IC: 6,33-6,77) e do grupo MedESF (6,51 e IC:6,23-6,79) (tabela 2).
Não houve diferença estatisticamente significativa na proporção de valores altos e baixos para o Escore Geral da APS avaliado para todo o país. Na região Nordeste, foi observada diferença significativa $(P=0,049)$ entre os grupos, com maior proporção de alto Escore Geral no grupo MMCuba $(58,8 \%)$ em comparação ao MMBrasil (51,8\%) e MedESF (52,1\%) (tabela 3). A proporção de alto Escore de Acesso no Brasil e nas regiões Norte, Nordeste e Centro-Oeste foi maior para o grupo MMCuba do que para os demais grupos de médicos (tabela 3).

$\mathrm{Na}$ análise univariável, as variáveis dos usuários associadas ao Escore Geral da APS foram: idade, estrato socioeconômico, presença de doenças crônicas, número de consultas na unidade e se o médico realiza visita domiciliar.
As variáveis dos médicos foram: sexo, tempo de formado em medicina e número médio de consultas por turno de 4 horas (tabela 4). As variáveis que permaneceram no modelo multivariável foram: idade do usuário (categorizada em tercis), estrato socioeconômico, presença de uma ou mais doenças crônicas (entre hipertensão, diabetes, depressão e doença pulmonar crônica), o médico realizar visita domiciliar e o tempo de formado do médico (tabela 4). A variável mais expressiva no modelo foi "o Dr. realiza visita domiciliar", que produziu um incremento significativo, de 1,17 ponto, no Escore Geral da APS (tabela 4). A variável "número de consultas na unidade nos últimos 12 meses" foi removida do modelo multivariável por apresentar colinearidade com a variável que expressa o perfil de doenças do usuário. 
TABELA 2. Escore Gerala, Escore de Acesso e Escore de Longitudinalidade ${ }^{a}$ conforme avaliação dos usuários adultos da Estratégia Saúde da Família que consultam com médicos cubanos e brasileiros participantes ou não do Programa Mais Médicos, Brasil, 2016

\begin{tabular}{|c|c|c|c|c|}
\hline \multirow{2}{*}{ Região geográfica } & \multirow{2}{*}{ Grupo do médicob } & \multicolumn{3}{|c|}{ Média (IC95\%) } \\
\hline & & Escore Geral da APS & Escore de Acesso & Escore de Longitudinalidade \\
\hline \multirow[t]{5}{*}{ Brasil } & Geral & 6,78 (6,71 a 6,84) & $4,24(4,18$ a 4,30$)$ & $7,43(7,37$ a 7,49$)$ \\
\hline & MMCuba & $6,86(6,75$ a 6,97$)$ & $4,43(4,32$ a 4,54$)$ & $7,38(7,27$ a 7,50$)$ \\
\hline & MMBrasil & $6,74(6,63$ a 6,85$)$ & $4,08(3,98 \text { a } 4,18)^{d}$ & $7,43(7,33$ a 7,53$)$ \\
\hline & MedESF & $6,73(6,60$ a 6,86$)$ & $4,20(4,09 \text { a } 4,32)^{d}$ & $7,48(7,36$ a 7,60$)$ \\
\hline & $P$-valor ${ }^{c}$ & 0,215 & $<0,001$ & 0,529 \\
\hline \multirow{3}{*}{ Norte } & MMBrasil & $6,63(6,36$ a 6,90$)$ & $3,83(3,50$ a 4,17$)$ & $7,17(6,85$ a 7,49$)$ \\
\hline & MedESF & $6,28(5,82$ a 6,74$)$ & $3,77(3,41$ a 4,13$)$ & $7,07(6,59$ a 7,54$)$ \\
\hline & $P$-valor ${ }^{c}$ & 0,33 & 0,057 & 0,379 \\
\hline \multirow[t]{2}{*}{ Nordeste } & Geral & $6,66(6,55$ a 6,76$)$ & $4,20(4,10$ a 4,31$)$ & $7,30(7,20$ a 7,39$)$ \\
\hline & MMCuba & $6,90(6,72$ a 7,09$)$ & $4,48(4,26$ a 4,71$)$ & $7,36(7,20$ a 7,53$)$ \\
\hline \multirow{4}{*}{ Sudeste } & MMCuba & $6,90(6,56$ a 7,24$)$ & $4,38(4,17$ a 4,58$)$ & $7,48(7,28$ a 7,69$)$ \\
\hline & MMBrasil & $6,72(6,46$ a 6,99$)$ & $4,24(4,01$ a 4,47$)$ & $7,45(7,21$ a 7,69$)$ \\
\hline & MedESF & $6,84(6,60$ a 7,08$)$ & $4,19(3,97$ a 4,42$)$ & $7,55(7,32$ a 7,78$)$ \\
\hline & $P$-valor ${ }^{\mathrm{c}}$ & 0,314 & 0,449 & 0,833 \\
\hline \multirow[t]{5}{*}{ Sul } & Geral & $7,14(6,95$ a 7,32$)$ & $4,36(4,20$ a 4,52) & $7,89(7,72$ a 8,06$)$ \\
\hline & MMCuba & $6,90(6,59$ a 7,21$)$ & $4,42(4,12$ a 4,72$)$ & $7,60(7,28$ a 7,92$)$ \\
\hline & MMBrasil & $7,18(6,87$ a 7,49$)$ & $4,24(4,00$ a 4,48$)$ & $8,04(7,78$ a 8,30$)$ \\
\hline & MedESF & $7,35(7,05$ a 7,64$)$ & $4,42(4,14$ a 4,70$)$ & $8,04(7,76$ a 8,32$)$ \\
\hline & $P$-valor ${ }^{c}$ & 0,146 & 0,525 & 0,075 \\
\hline \multirow[t]{2}{*}{ Centro-0este } & Geral & $6,68(6,38$ a 6,98$)$ & $4,35(4,01$ a 4,69$)$ & $7,41(7,11$ a 7,70$)$ \\
\hline & MMCuba & $6,71(6,08$ a 7,34$)$ & $4,5(3,76$ a 5,25$)$ & $7,56(6,89$ a 8,23$)$ \\
\hline
\end{tabular}

a Escore Geral obtido pela aplicação da PCATool-Brasil versão reduzida e Escore de Acesso e Longitudinalidade obtidos pelos itens dos atributos presentes no PCATool- Brasil versão expandida; varia de 0 a 10 . Escores $\geq 6,6$ são classificados como altos.

${ }^{b}$ MMCuba: médicos cubanos participantes do Programa Mais Médicos; MMBrasil: médicos brasileiros participantes do Programa Mais Médicos; MedESF: médicos brasileiros não participantes do Programa Mais Médicos.

c Associado à análise de variância (ANOVA).

${ }^{\mathrm{d}} P$-valor $<0,05$ na comparação múltipla de Bonferroni, considerando MMCuba como categoria de referência.

TABELA 3. Proporção de escores altos e baixos conforme avaliação de usuários adultos da Estratégia Saúde da Família atendidos por médicos cubanos e brasileiros participantes ou não do Programa Mais Médicos, Brasil, 2016

\begin{tabular}{|c|c|c|c|c|c|c|c|c|c|c|}
\hline \multirow[b]{2}{*}{ Região } & \multirow{2}{*}{$\begin{array}{l}\text { Grupo do } \\
\text { médico }^{b}\end{array}$} & \multicolumn{3}{|c|}{ Escore Geral da APS } & \multicolumn{3}{|c|}{ Escore de Longitudinalidade $^{a}$} & \multicolumn{3}{|c|}{ Escore de Acesso ${ }^{a}$} \\
\hline & & $\begin{array}{l}\text { Baixo } \\
\text { n (\%) }\end{array}$ & $\begin{array}{c}\text { Alto } \\
\mathrm{n}(\%)\end{array}$ & $P$-valor ${ }^{c}$ & $\begin{array}{l}\text { Baixo } \\
\text { n (\%) }\end{array}$ & $\begin{array}{c}\text { Alto } \\
\mathrm{n}(\%)\end{array}$ & $P$-valor ${ }^{c}$ & $\begin{array}{l}\text { Baixo } \\
\text { n (\%) }\end{array}$ & $\begin{array}{c}\text { Alto } \\
\mathrm{n}(\%)\end{array}$ & $P$-valor ${ }^{c}$ \\
\hline \multirow[t]{2}{*}{$\overline{\text { Brasil }}$} & MMCuba & $867(41,7)$ & $1212(58,3)$ & 0,643 & $623(29,9)$ & $1463(70,1)$ & 0,812 & $1870(90,4)$ & $199(9,6)$ & 0,001 \\
\hline & MedESF & $865(43,1)$ & $1140(56,9)$ & & $576(28,6)$ & $1435(71,4)$ & & $1859(93,2)$ & $135(6,8)^{d}$ & \\
\hline \multirow[t]{2}{*}{ Norte } & MMCuba & $138(54,8)$ & $114(45,2)$ & 0,241 & $119(47,4)$ & $132(52,6)$ & 0,110 & $233(92,8)$ & $18(7,2)$ & 0,020 \\
\hline & MMBrasil & $110(45,3)$ & $133(54,7)$ & & $80(32,7)$ & $165(67,3)$ & & $233(97,5)$ & $6(2,5)^{d}$ & \\
\hline \multirow[t]{3}{*}{ Nordeste } & MMCuba & $306(41,2)$ & $436(58,8)$ & 0,049 & $220(29,5)$ & $525(70,5)$ & 0,233 & $662(90,2)$ & $72(9,8)^{\mathrm{e}}$ & 0,020 \\
\hline & MMBrasil & $366(48,2)$ & $393(51,8)^{d}$ & & $257(33,8)$ & $504(66,2)$ & & $713(94,7)$ & $40(5,3)^{\mathrm{d}}$ & \\
\hline & MedESF & $329(47,9)$ & $358(52,1)^{d}$ & & $230(33,4)$ & $458(66,6)$ & & $629(91,7)$ & $57(8,3)^{\mathrm{e}}$ & \\
\hline
\end{tabular}


TABELA 3. (Continuação)

\begin{tabular}{|c|c|c|c|c|c|c|c|c|c|c|}
\hline \multirow[b]{2}{*}{ Região } & \multirow{2}{*}{$\begin{array}{c}\text { Grupo do } \\
\text { médico }^{\text {b }}\end{array}$} & \multicolumn{3}{|c|}{ Escore Geral da APS ${ }^{a}$} & \multicolumn{3}{|c|}{ Escore de Longitudinalidade ${ }^{a}$} & \multicolumn{3}{|c|}{ Escore de Acesso $^{a}$} \\
\hline & & $\begin{array}{l}\text { Baixo } \\
\mathrm{n}(\%)\end{array}$ & $\begin{array}{c}\text { Alto } \\
\mathrm{n}(\%)\end{array}$ & $P$-valor & $\begin{array}{l}\text { Baixo } \\
\text { n (\%) }\end{array}$ & $\begin{array}{c}\text { Alto } \\
\mathrm{n}(\%)\end{array}$ & $P$-valor ${ }^{c}$ & $\begin{array}{l}\text { Baixo } \\
\mathrm{n}(\%)\end{array}$ & $\begin{array}{c}\text { Alto } \\
\mathrm{n}(\%)\end{array}$ & $P$-valor ${ }^{c}$ \\
\hline \multirow[t]{3}{*}{ Sudeste } & MMCuba & $217(36,4)$ & $379(63,6)$ & 0,076 & $154(25,8)$ & $443(74,2)$ & 0,471 & $540(91,4)$ & $51(8,6)$ & 0,080 \\
\hline & MMBrasil & $259(44,1)$ & $328(55,9)$ & & $171(29,0)$ & $418(71,0)$ & & $534(91,8)$ & $48(8,2)$ & \\
\hline & MedESF & $256(40,2)$ & $381(59,8)$ & & $163(25,5)$ & $477(74,5)$ & & $600(94,9)$ & $32(5,1)$ & \\
\hline \multirow[t]{3}{*}{ Sul } & MMCuba & $143(39,6)$ & $218(60,4)$ & 0,077 & $93(25,8)$ & $268(74,2)$ & 0,044 & $323(89,5)$ & $38(10,5)$ & 0,480 \\
\hline & MMBrasil & $109(32,6)$ & $225(67,4)$ & & $55(16,5)$ & $279(83,5)^{d}$ & & $302(91,8)$ & $27(8,2)$ & \\
\hline & MedESF & $101(29,2)$ & $245(70,8)$ & & $59(17,0)$ & $289(83,0)^{d}$ & & $310(89,3)$ & $37(10,7)$ & \\
\hline \multirow[t]{3}{*}{ Centro-Oeste } & MMCuba & $63(49,2)$ & $65(50,8)$ & 0,236 & $37(28,0)$ & $95(72,0)$ & 0,708 & $112(84,8)$ & $20(15,2)$ & 0,010 \\
\hline & MMBrasil & $50(37,6)$ & $83(62,4)$ & & $38(28,6)$ & $95(71,4)$ & & $128(97,0)$ & $4(3,0)^{d}$ & \\
\hline & MedESF & $57(51,8)$ & $53(48,2)$ & & $37(33,6)$ & $73(66,4)$ & & $104(97,2)$ & $3(2,8)^{d}$ & \\
\hline
\end{tabular}

a Alto $\geq 6,6$ pontos; baixo $<6,6$ pontos.

b MMCuba: médicos cubanos participantes do Programa Mais Médicos; MMBrasil: médicos brasileiros participantes do Programa Mais Médicos; MedESF: médicos brasileiros não participantes do Programa Mais Médicos.

${ }^{c}$ Associado a estatística de teste do qui-quadrado.

d $P$-valor <0,05, associado à comparação múltipla, sendo MMCuba a categoria de referência.

e $P$-valor <0,05, associado à comparação múltipla, sendo MMBrasil a categoria de referência.

TABELA 4. Modelo univariável e multivariável das características associadas ao Escore Geral da APS na Estratégia Saúde da Família por meio da metodologia de análise multinível, Brasil, 2016

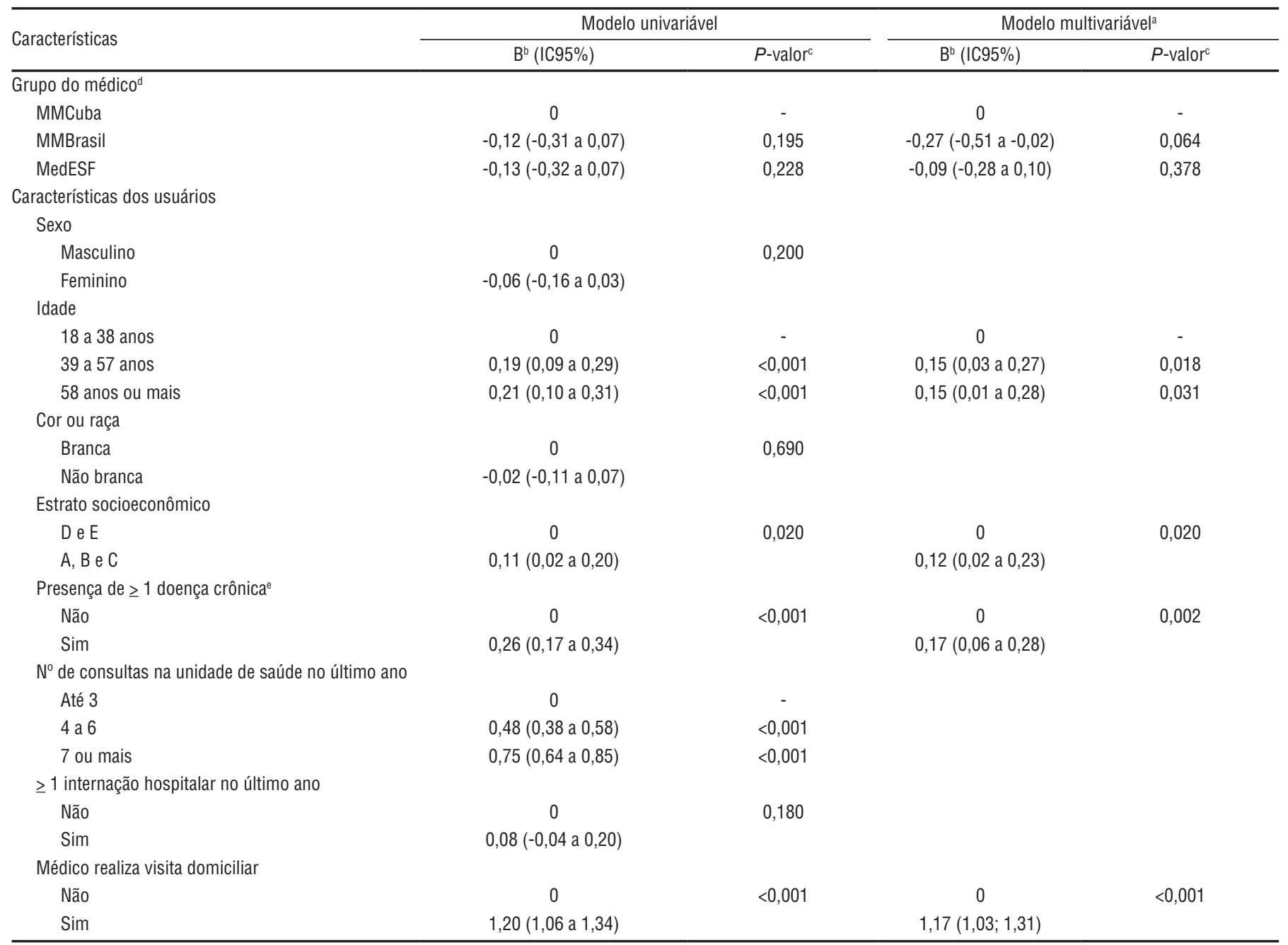


TABELA 4. (Continuação)

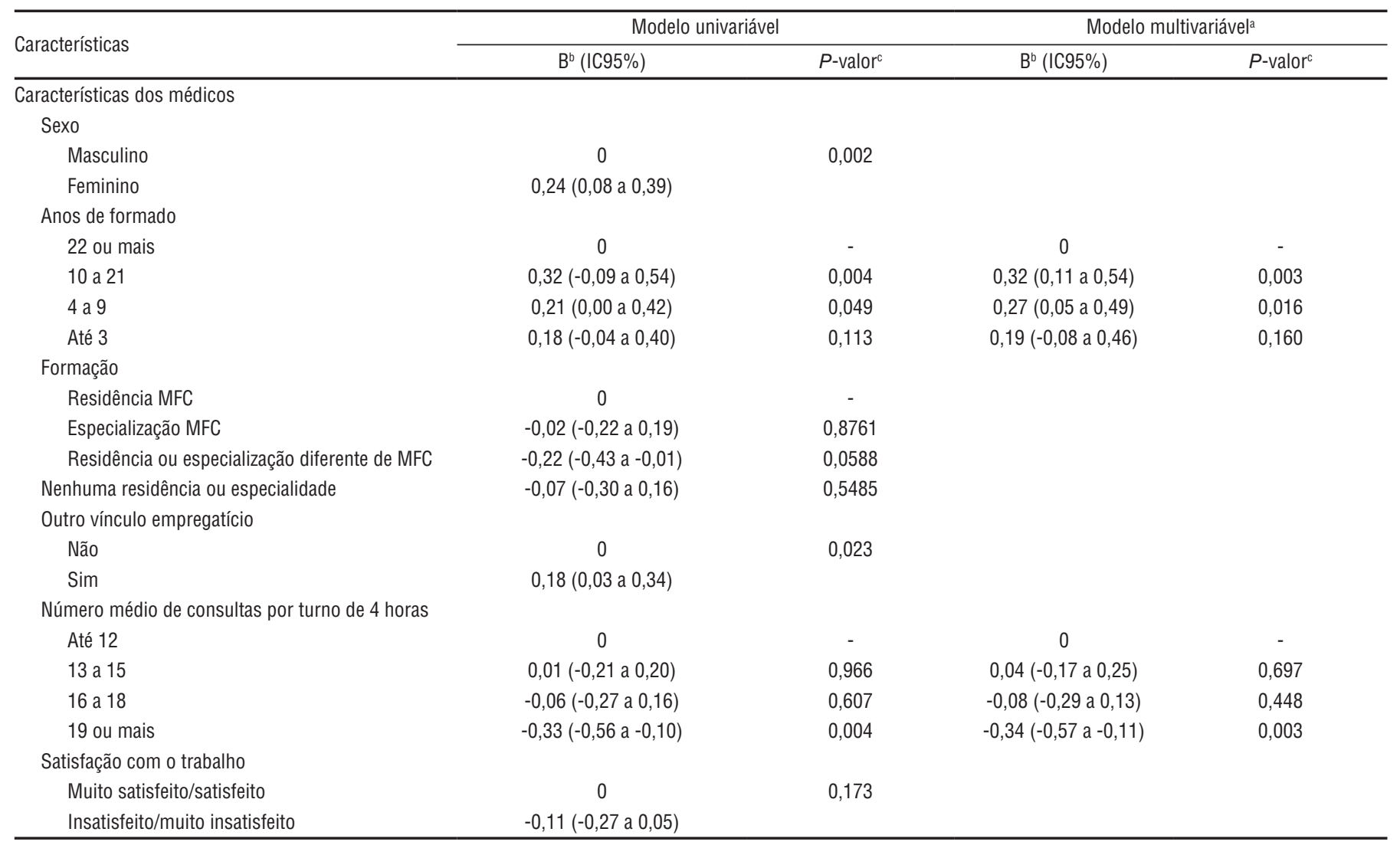

a Variáveis que se mantiveram no modelo final.

${ }^{\mathrm{b}} \mathrm{B}$ : coeficiente beta do modelo.

${ }^{\mathrm{c}}$ Associado ao modelo multinível de efeito aleatório.

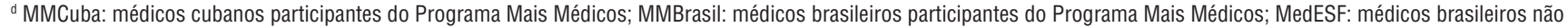
participantes do Programa Mais Médicos.

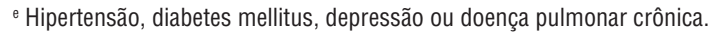

\section{DISCUSSÃO}

O presente estudo mostrou que o provimento emergencial de médicos do PMM associou-se a um pequeno aumento no Escore de Acesso obtido com o PCATool-Brasil, com maior proporção de alto escore em regiões de maior vulnerabilidade socioeconômica (Norte, Nordeste e Centro-Oeste). O Escore Geral da APS e seu componente Longitudinalidade ultrapassaram o ponto de corte para alto escore, porém com valores ainda muito próximos do limite, demonstrando um amplo espaço para fortalecimento da APS no país. Vale notar que os resultados mostraram um avanço discreto na qualidade da APS nos últimos anos se comparado o Escore Geral com aquele medido com o instrumento PCATool-Brasil em estudos anteriores, de âmbito local (1014). Foi possível identificar que a atuação do médico - número de consultas e realização de visitas domiciliares - teve mais influência no Escore Geral do que sua especialização. A presença de doenças crônicas, idade mais avançada e estrato socioeconômico A, B e C dos usuários também influenciaram positivamente o Escore Geral da APS. Importante salientar que a nacionalidade do médico, cubana ou brasileira, não apresentou associação de magnitude importante com os escores de APS medidos, ou seja, mesmo na presença de diferenças significativas a diferença no valor numérico não era tão relevante.

Foram pontos fortes deste estudo o seu caráter de avaliação objetiva da APS brasileira, conforme seus atributos definidores, e a utilização de uma amostra grande (6 160 pessoas entrevistadas de 6193 necessárias). Entre as limitações do estudo, o Escore de Acesso e Longitudinalidade podem estar superestimados, pois a coleta foi realizada com população que já consultava na unidade (suplantando outras barreiras de acesso) e com médico que atuava há pelo menos 1 ano no local (não expressando a realidade de unidades com maior rotatividade profissional). Outra limitação relaciona-se à avaliação do efeito da especialização sobre o Escore Geral da APS, já que o processo de formação médica é diverso entre Brasil e Cuba, não sendo avaliado detalhadamente por este estudo.

Uma revisão sistemática de estudos realizados com o PCATool-Brasil evidenciou heterogeneidade da APS, com variação na amplitude do Escore Geral obtido em diversos municípios (10). Harzheim et al. e Oliveira et al. identificaram maior fragilidade especialmente nos componentes de Acesso e Integralidade (serviços prestados) $(11,12)$. Em concordância com essas pesquisas, este estudo mostra que o Escore de Acesso continua baixo em todo o país. Nas 
regiões Norte, Nordeste e Centro-Oeste, que apresentam maior vulnerabilidade socioeconômica e escassez de médicos, as proporções de alto Escore de Acesso foram maiores no grupo MMCuba em relação aos demais grupos. Isso representa um discreto avanço no alcance da equidade, objetivo da APS, definido por Starfield $(6,7,21,27)$. O aumento no Escore de Acesso sugere fortalecimento desse atributo nessas regiões. Contudo, a análise multivariável mostrou que usuários pertencentes aos estratos econômicos D e E diminuíam a pontuação do Escore Geral, mostrando uma potencial persistência na fragilidade do cuidado oferecido a esse perfil de população.

A APS no Brasil possui grandes dificuldades para prover acesso de fato aos usuários. Estudos mostram que o PMM tem contribuído para o aumento da assistência médica no país, também identificada pela redução de municípios com escassez de médicos $(4,7)$. No presente estudo, a média de consultas médicas na unidade de saúde no ano anterior ao estudo foi quase o dobro da média nacional de consultas/habitante/ano (15). O resultado, contudo, não reflete necessariamente aumento no acesso a consultas para toda população, podendo estar associado à maior utilização por parte dos usuários que já consultam na unidade, com realização de revisões frequentes, no caso de pessoas com doenças crônicas estáveis.

Estudo nacional de Fausto et al. identificou dificuldades no acesso para marcação de consultas - apenas $28 \%$ dos usuários conseguiam agendar consulta a qualquer momento e 30,8\% pegavam fichas e faziam fila antes da unidade abrir - e para atendimento de urgências/emergências - 35\% dos usuários não buscaram a unidade pois estava fechada (17). Em nosso estudo, quase metade das consultas realizadas no dia da entrevista foi obtida naquele mesmo dia. Mesmo assim, um terço dos usuários referiu que era difícil conseguir atendimento quando necessário e menos de $10 \%$ identificavam que suas unidades prestavam atendimento em horário estendido. Em países com APS forte, estratégias para melhorar o acesso são realizadas com a ampliação do horário de atendimento para além dos horários convencionais. É o caso do Canadá e do Reino Unido, que oferecem atendimento em APS 24 horas por dia e por meio de telessaúde, com canal telefônico para fornecer orientações de saúde, ou da
Espanha, que mantém turnos estendidos até as 21 horas (28-30).

Observamos que, embora os médicos do PMM estivessem há menos tempo nas unidades, o Escore de Longitudinalidade não foi inferior para esses profissionais. $\mathrm{O}$ vínculo estabelecido com o serviço, baseado no território, pode ser um fator facilitador desse processo. Conceitualmente, a longitudinalidade é definida como uma relação pessoal e de longa duração entre os profissionais de saúde e usuários do serviço, que serve como referência para as pessoas, independentemente da presença de problemas específicos de saúde (27). Países como Canadá, Reino Unido e Austrália têm optado por fortalecer a longitudinalidade pelo vínculo do usuário com um médico de família e comunidade de sua escolha, não necessariamente próximo à sua residência, que é responsável por uma lista restrita de pacientes com cerca de 1500 usuários $(28,29,31)$. No Brasil, a obrigatoriedade de atendimento em território e a responsabilidade da unidade pelo atendimento de cerca de 3000 pessoas podem estar prejudicando a melhora da longitudinalidade e do acesso.

$\mathrm{Na}$ análise multinível, a especialidade do médico não apresentou relevância significativa para o Escore Geral da APS. Vale ressaltar, porém, que a amostra não foi calculada para medir essa associação. Castro et al. e Chomatas et al., por sua vez, encontraram associação positiva entre possuir especialização em APS e alto Escore Geral $(13,14)$. As evidências internacionais também apontam nessa direção, mostrando melhores resultados em saúde onde há maior densidade de médicos de família e comunidade por habitante em comparação a outros especialistas $(27,32)$. Apesar da ausência de associação com a especialidade, a realização de visita domiciliar, uma das tarefas intrínsecas de um médico da APS, teve associação direta com maior pontuação no Escore Geral (33-35).

Embora o PMM tenha proporcionado aumento de médicos especialistas em APS, limitações de estrutura locais e da rede de atenção podem minimizar o potencial de eficiência e resolutividade das ações dos médicos de família e comunidade (32). Giovanella et al. identificaram essa heterogeneidade na estrutura ao estudar 38812 unidades de saúde no país, pois somente $4,8 \%$ apresentavam todas as dimensões necessárias para seu melhor funcionamento e $44,1 \%$ não tinham equipamentos e insumos básicos suficientes (4). Um estudo sobre a infraestrutura das unidades de saúde que receberam médicos do PMM apontou que 62,5\% delas apresentavam infraestrutura de média qualidade e 5,8\% de baixa qualidade (5).

A heterogeneidade na qualidade da APS no Brasil pode ser explicada, entre diversos outros fatores de estrutura e gestão, pela dificuldade de fixação de médicos em unidades de saúde, especialmente em regiões mais remotas, e ao número reduzido de médicos com formação especializada para trabalhar na APS $(7,18)$. Esses dois fatores fragilizam a qualidade do cuidado médico, limitando a sua potencial efetividade e reduzindo o grau de resolutividade clínica das equipes. Contudo, a qualidade da APS de um país é também influenciada por elementos de estrutura, como instalações, equipamentos, organização da rede de atenção, que agem hierarquicamente sobre os processos (atributos definidores) e resultados de saúde $(29,32)$.

\section{CONCLUSÕES}

Este estudo mostrou que a estratégia nacional para alocação de médicos está associada a maior Escore de Acesso, principalmente em regiões de maior vulnerabilidade socioeconômica e com escassez de médicos. Pode-se afirmar que o tipo de médico, cubano ou brasileiro, não influenciou diferentemente o grau de orientação (Escore Geral) da APS no país. O Escore Geral da APS no país e o componente Longitudinalidade ultrapassaram levemente o ponto de corte para a categorização de um alto escore, apontando a necessidade de novas intervenções para fortalecer a APS no Brasil, como reforço dos papéis fundamentais do médico (visitas domiciliares em situações necessárias) e aprimoramento do acesso das populações de maior vulnerabilidade socioeconômica e de pessoas mais jovens e sem problemas de saúde estabelecidos. Novos estudos são importantes para avaliar elementos de estrutura (qualidade técnica dos profissionais, equipamentos disponíveis, entre outros) e processo (analisando os componentes do PCATool-Brasil com sua versão expandida) que possam influenciar na qualidade da APS brasileira.

Agradecimentos. Agradecemos à Organização Pan-Americana da Saúde/ Organização Mundial da Saúde (OPAS/ OMS) pelo financiamento que possibilitou a coleta de dados e a execução do projeto e ao TelessaúdeRS-UFRGS pelo apoio logístico. 
Conflitos de interesse. Estudo proposto por pesquisadores do Programa de Pós-Graduação em Epidemiologia, da Faculdade de Medicina da Universidade Federal do Rio Grande do Sul (UFRGS), e financiado pela
Organização Pan-Americana de Saúde/Organização Mundial da Saúde (OPAS/OMS). Os autores declaram ter avaliado os dados conforme evidências, não havendo conflito de interesses.

\section{REFERÊNCIAS}

1. Brasil, Ministério da Saúde. Política Nacional de Atenção Básica. Brasília: Ministério da Saúde; 2012. Disponível em: http:/ /189.28.128.100/dab/docs/publicacoes/geral/pnab.pdf Acessado em 28 de julho de 2018.

2. Brasil, Ministério da Saúde. Portaria 2 436/2017. Diário Oficial da União 2017;22 set;Seção 1:68. Disponível em: http:/ / pesquisa.in.gov.br/imprensa/jsp/ visualiza/index.jsp?jornal $=1 \&$ pagina $=$ $68 \&$ data $=22 / 09 / 2017$ Acessado em 28 de julho de 2018.

3. Brasil, Ministério da Saúde. Histórico de cobertura da Estratégia Saúde da Família. Brasília: Ministério da Saúde; 2017. Disponível em: http://dab.saude.gov.br/ portaldab/historico_cobertura_sf.php Acessado em 28 de julho de 2018.

4. Giovanella L, Mendonça MH, Fausto MC, Almeida PF, Bousquat A, Lima JG, et al. Emergency supply of doctors by the Mais Médicos (More Doctors) Program and the quality of the structure of primary health care facilities. Cienc Saude Colet. 2016;21(9):2697-708.

5. Giovanella L, Bousquat A, Fausto MCCR, Fusaro RE, Mendonça MHM, Gagno J. Novos caminhos: tipologia das unidades básicas de saúde brasileiras. Nota Técnica 5/2015. Região e Redes: Caminho da Universalização da Saúde da Saúde no Brasil; 2015. Disponível em: http://www. resbr.net.br/wp-content/uploads / 2015/09/NovosCaminhos05_ValeEste. pdf Acessado em 28 de julho de 2018.

6. Girardi SN, editor. Identificação de áreas de escassez em recursos humanos em saúde: relatório final de atividades. Belo Horizonte: Universidade Federal de Minas Gerais; 2012. Disponível em: http:/ / epsm.nescon.medicina.ufmg.br/ epsm/Relate_Pesquisa / Identifica \% C $3 \%$ A $7 \%$ C $3 \%$ A $30 \% 20$ de $\% 20 \%$ C $3 \%$ A 1 reas $\% 20$ de $\% 20$ escassez $\% 20$ de $\% 20$ RHS\%20no\%20Brasil.pdf Acessado em 28 de julho de 2018.

7. Girardi SN, Stralen ACS, Cella JN, Wan Der Maas L, Carvalho CL, Faria EO. Impact of the Mais Médicos (More Doctors) Program in reducing physician shortage in Brazilian Primary Healthcare. Cienc Saude Colet. 2016;21(9):2675-684.

8. Trindade TG. Associação entre extensão dos atributos de atenção primária e qualidade do manejo da hipertensão arterial em adultos adscritos à rede de atenção primária à saúde de Porto Alegre [dissertação]. Porto Alegre: Universidade Federal do Rio Grande do Sul; 2007. Disponível em: http://hdl.handle.net/
10183/12947 Acessado em 28 de julho de 2018.

9. Gonçalves MR. Associação entre a qualidade da atenção primária à saúde e o processo de atenção aos portadores de diabetes mellitus adscritos aos serviços de saúde em Porto Alegre [dissertação]. Porto Alegre: Universidade Federal do Rio Grande do Sul; 2007. Disponível em: http:/ / hdl.handle.net/10183/13199 Acessado em 28 de julho de 2018.

10. D'Avila OP, Pinto LF, Hauser L, Gonçalves MR, Harzheim E. The use of the Primary Care Assessment Tool (PCAT): an integrative review and proposed update. Cienc Saude Colet. 2017;22(3):855-65.

11. Harzheim E, Pinto LF, Hauser L, Soranz D. Assessment of child and adult users of the degree of orientation of Primary Healthcare in the city of Rio de Janeiro, Brazil. Cienc Saude Colet. 2016;21(5): 1399-408.

12. Oliveira MMC. Presença e extensão dos atributos da atenção primária à saúde entre os serviços de atenção primária em Porto Alegre: uma análise agregada [dissertação]. Porto Alegre: Universidade Federal do Rio Grande do Sul; 2007. Disponível em: http://hdl.handle.net/ 10183/12649 Acessado em 28 de julho de 2018.

13. Castro RCL, Knauth DR, Harzheim E, Hauser L, Duncan BB. Avaliação da qualidade da atenção primária pelos profissionais de saúde: comparação entre diferentes tipos de serviços. Cad Saude Publica. 2012;28(9):1772-84.

14. Chomatas E, Vigo A, Marty I, Hauser L, Harzheim E. Avaliação da presença e extensão dos atributos da atenção primária em Curitiba. Rev Bras Med Fam Comunidade. 2013;8(29):294-303.

15. Brasil, Ministério da Saúde. IBD 2012 Brasil. Indicadores de cobertura: número de consultas médicas (SUS) por habitante. Brasília: Ministério da Saúde; 2013. Disponível em: http://tabnet.datasus. gov.br/cgi/tabcgi.exe?idb2012/f01.def Acessado em 28 de julho de 2018.

16. Organisation for Economic Co-operation and Development. OECD statistics: health care utilisation. Paris: OECD; 2018. Disponível em: http://stats.oecd.org/ Index.aspx? DataSetCode=HEALTH PROC Acessado em 28 de Julho de 2018.

17. Fausto MCR, Giovanella L, Mendonça MHM, Seidl H, Gagno J. A posição da Estratégia Saúde da Família na rede de atenção à saúde na perspectiva das equipes e usuários participantes do PMAQAB. Saude Debate. 2014;38:13-33.
Declaração. As opiniões expressas no manuscrito são de responsabilidade exclusiva dos autores e não refletem necessariamente a opinião ou política da RPSP/ PAJPH ou da Organização PanAmericana da Saúde (OPAS).
18. Scheffer M, editor. Demografia médica no Brasil 2015. São Paulo: Departamento de Medicina Preventiva da Faculdade de Medicina da USP; 2015. Disponível em: http:/ / www.usp.br/agen/wp-content/ uploads/DemografiaMedica30nov2015. pdf Acessado em 28 de julho de 2018.

19. World Health Organization (WHO). Global Health Observatory (GHO) data. Density of physicians (total number per 1000 population, latest available year) and density per 1000 data by country. Genebra: WHO; 2017. Disponível em: http://www.who. int/gho/health_workforce/physicians_ density/en/ Acessado em 29 de julho de 2018.

20. Brasil. Lei 12 871/2013. Diário Oficial da União. 2013;23 out; Seção 1:1. Disponível em: http://www.planalto.gov.br/ccivil_ 03/_Ato2011-2014/2013/Lei/L12871.htm Acessado em setembro de 2018.

21. Oliveira JPA, Sanchez MN, Santos LMP. The Mais Médicos (More Doctors) Program: the placement of physicians in priority municipalities in Brazil from 2013 to 2014. Cienc Saude Colet. 2016;21(9): 2719-27.

22. Brasil, Ministério da Saúde. Nota Técnica ${ }^{\circ}$ 193/2018-CGPS/DEPREPS/SGTES/MS. Brasília: Ministério da Saúde; 2018.

23. Harzheim E, Oliveira MMC, Agostinho MR, Hauser L, Stein AT, Gonçalves MR, et al. Validação do instrumento de avaliação da atenção primária à saúde: PCAToolBrasil adultos. Rev Bras Med Fam Comunidade. 2013:8(29):274-84

24. Oliveira MMC, Harzheim E, Riboldi J, Duncan BB. PCATool-ADULTO-BRASIL: uma versão reduzida. Rev Bras Med Fam Comunidade. 2013:8(29):256-63.

25. Zar JH. Biostatistical analysis. New Jersey: Prentice Hall; 1999.

26. Brown H, Prescott R. Applied mixed models in models. $2^{\text {a }}$ ed. Chichester: John Wiley \& Sons; 2006.

27. Starfield, B. Atenção primária: equilíbrio entre necessidades de saúde, serviços e tecnologia. Brasília: Organização das Nações Unidas para a Educação, a Ciência e a Cultura/Ministério da Saúde; 2002.

28. Marchildon GP. Canada: Health system review. Health Syst Transit. 2013;15(1): 1-179.

29. Cylus J, Richardson E, Findley L, Longley M, O'Neill C, Steel D. United Kingdom: health system review. Health Syst Transit. 2015;17(5):1-125.

30. García-Armesto S, Abadía-Taira MB, Durán A, Hernández-Quevedo C, BernalDelgado E. Spain: health system review. Health Syst Transit. 2010;12(4):1-295. 
31. Australian Institute of Health and Welfare. Australia's health 2016. Canberra: AIHW; 2016. Disponível em: http://www.aihw. gov.au/WorkArea/DownloadAsset.aspx? id $=60129555788$ Acessado em 29 de julho de 2018.

32. Kringos DS, Boerma WGW, Hutchinson A, Saltman RB, editores. Building primary care in a changing Europe. Londres: The European Observatory on Health Systems and Policies; 2015. Disponível em: http:// www.euro.who.int/__data/assets/pdf_ file / 0018/271170/BuildingPrimary CareChangingEurope.pdf Acessado em setembro de 2018.
33. Trindade TG, Batista SR. Family and community medicine: now more than ever! Cienc Saude Colet. 2016;21(9):2667-9.

34. Wonca Europe. The European definition of general practice / family medicine. Barcelona: WONCA Europe; 2011. Disponível em: http:/ / www.woncaeurope.org/ sites/default/files/documents/Definition\% 203rd\%20ed\%202011\%20with\%20revised\% 20wonca\%20tree.pdf Acessado em 29 de julho de 2018.

35. Arias-Castillo L, Toro CB, Freifer S, Fernández MA. Perfil do médico de família e comunidade definição iberoamericana. Santiago de Cali: Confederação
Iberoamericana de Medicina de Família e Comunidade; 2010. Disponível em: http://www.sbmfc.org.br/media/file/ documentos/perfil_mfc.pdf Acessado em 29 de julho de 2018.

Manuscrito recebido em 13 de janeiro de 2018. Aceito em versão revisada em 2 de agosto de 2018.
ABSTRACT

Primary health care performance in Brazil and association with the More Doctors physician recruitment program
Objective. To assess the performance of primary health care (PHC) in Brazil and its association with the More Doctors Program (Programa Mais Médicos, PMM).

Method. This nationwide cross-sectional study used the Primary Care Assessment Tool validated for Brazilian Portuguese (PCATool-Brasil) to determine the achievement of PHC according to user experience associated with three physician categories: Brazilian physicians participating in the PMM, Cuban physicians participating in the PMM, and Brazilian physicians not linked to the PMM. The following PHC scores were calculated: overall PCA score, accessibility (first contact), and longitudinality. The association between PHC scores, physician category, and other user and physician characteristics was investigated using multilevel analysis.

Results. The overall PCA score for Brazil was 6.78, and the longitudinality score was 7.43. There was no difference in these scores among the three physician categories. The overall accessibility score was 4.24. A small but significant difference $(P<0.001)$ in accessibility score was detected among physician categories: 4.43 for Cuban physicians participating in the PMM (CI: 4.32-4.54), 4.08 for Brazilian physicians participating in the PMM(CI: 3.98-4.18), and 4.20 for Brazilian physicians not linked to the PMM (CI: 4.09-4.32). Age, socioeconomic level, presence of chronic diseases, and home visits by physicians positively influenced the overall PCA score on multilevel analysis.

Conclusions. The type of physician did not influence the primary care orientation (overall score) of the healthcare system in Brazil. PMM was associated with higher accessibility sores in more socioeconomically vulnerable areas. Multilevel analysis showed that PCH may be strengthened by the reinforcement of essential physician roles (such as home visits) and by improving access for socioeconomically vulnerable, younger populations or those without chronic diseases.

Keywords Primary health care; Family Health Strategy; health services research; Unified Health System; health systems; Brazil. 
RESUMEN

Calidad de la atención primaria de salud en Brasil y relación con el Programa Más Médicos

Objetivo. Evaluar la calidad de la atención primaria de salud (APS) en Brasil y su relación con el Programa Más Médicos (PMM).

Métodos. Estudio transversal de alcance nacional em el que se utilizó la herramienta PCATool-Brasil para evaluar la calidad de la APS a partir de la experiencia de los usuarios vinculados a tres categorías de médicos: médicos brasileños del PMM, médicos cubanos del PMM y médicos brasileños no vinculados al PMM. Se calcularon los siguientes puntajes: Puntaje general de APS, Puntaje de acceso y Puntaje de longitudinalidad. Se investigaron mediante análisis multinivel la asociación entre el Puntaje general obtenido, la categoría del médico y otras características de los usuarios y los profesionales.

Resultados. El Puntaje general de APS para Brasil fue 6,78, y el Puntaje de longitudinalidad, 7,43. No hubo diferencia entre estos puntajes para las tres categorías de médicos. El Puntaje de acceso para Brasil fue de 4,24 y mostró una diferencia pequeña, pero significativa ( $\mathrm{p}<0,001)$ entre las categorías de médicos: médicos cubanos del PMM 4,43 (IC: 4,32-4,54), médicos brasileños del PMM 4,08 (IC: 3,98-4,18) y médicos brasileños no vinculados al PMM 4,20 (IC: 4,09-4,32). En el análisis multinivel, la edad, el estrato socioeconómico, la presencia de enfermedades crónicas y el hecho de que el médico realizara visitas domiciliarias influyeron positivamente em el Puntaje general.

Conclusiones. El tipo de médico no influyó en el grado de orientación a los atributos de la APS (Puntaje general) en Brasil. El PMM se asoció com mayores Puntajes de acceso en las regiones de mayor vulnerabilidad socioeconómica. Con el análisis multinivel se identificó que el fortalecimiento de la APS también puede ser alcanzado reforzando los roles fundamentales de los médicos que trabajan en la APS (como las visitas domiciliarias) y mejorando el acceso de las poblaciones de mayor vulnerabilidad socioeconómica y de las personas más jóvenes o sin enfermedades crónicas.

Palabras clave

Atención primaria de salud; Estrategia de Salud Familiar; investigación en servicios de salud; Sistema Único de Salud; sistemas de salud; Brasil. 